\title{
A Distributed Dynamic Bandwidth Allocation Algorithm in EPON
}

\author{
Feng Cao, Deming Liu, Minming Zhang, Kang Yang \& Yinbo Qian \\ School of Optoelectronic Science and Engineering \\ Huazhong University of Science and Technology, Wuhan 430074, China \\ National Engineering Laboratory for Next Generation Internet Access System, Wuhan 430074, China \\ Wuhan National Laboratory for Optoelectronics, Wuhan 430074, China \\ E-mail:hellocaofeng@gmail.com
}

\begin{abstract}
EPON (Ethernet Passive Optical Network) is a rising bandwidth access technology, and it could realize the comprehensive operation access including data, video, and voice, with good economic characters. IEEE 802.3ah is the industrial standard of EPON, but it doesn't concretely regulate the uplink bandwidth allocation algorithm of EPON. Therefore, aiming at the uplink channel access of EPON, people have put forward various dynamic bandwidth allocation algorithms, but most of them belong to intensive algorithm, i.e. the distributed bandwidth allocation (DBA) algorithm runs in OLT which is the interceder to allocate the uplink transmission time slot for ONU. A new distributed dynamic bandwidth allocation algorithm (DDBA) is proposed in this article, in which ONU decides the size of transmission window based on the assistant information transmitted by OLT and self queue length. The simulation result indicates that comparing with IPACT (Interleaved Poling with Adaptive Cycle Time) (G. Kramer, 2002, P.89-107), under the high network load, DDBA could obviously improve the average end-to-end time delay and the average queue length.
\end{abstract}

Keywords: EPON, DBA, Distributed scheduling, Access network (AN)

\section{Introduction}

With the occurrence of VOIP (voice of internet phone), video conference, and continually increasing data operation, new requirements occur for the bandwidth of network. But the bottleneck of the existing network bandwidth is in the access network, because the mainstream bandwidth access mode is the technology of DSL. The DSL technology developed based on the original phone network, and it doesn't fit for the transmission of data operation. PON is thought as one of the most hopeful technical projects to solve this bandwidth access bottleneck, and EPON adopting the Ethernet protocol has larger competitive advantage, and it begins to be produced in the market. EPON could realize the comprehensive operation access of data, voice, and video, with good economic character. Insiders generally think that FTTH is the final solution of the bandwidth access, but EPON will be a mainstream bandwidth access technology. Because of the characters of EPON network structure, the special advantage of bandwidth access, and the natural organic combination with the computer network, all experts in the whole world think that EPON is the optimal transmission medium to realize "three networks in one" and solve the "final one kilometer" of the info-highway.

EPON adopts the point-to-point network frame. The direction from OLT to ONU is the downlink direction, and OLT broadcasts, and ONU selectively accept the information according to the intention address of frame. The direction from ONU to OLT is the uplink direction, and there is only one uplink channel, and the data transmitted by different ONUs will conflict. To void that the frames sent by various ONUs conflict, a mechanism needs to harmonize the conflict induced by various ONUs transmit data to OLT. IEEE 802.3an standard defines multi-point control protocol (MPCP), and it defines five control frames for the login, the bandwidth request, and the bandwidth authorization of ONU. Where, REPORT frame is the bandwidth request frame that ONU transmits to OLT, and it is used to report the length of the ONU buffer queue. GATE frame is the bandwidth authorization frame that OLT transmits to ONU, and it is used to report when ONU transmits data. But the standard didn't concretely define which scheduling algorithm will exceed the standard range when it is used to allocate the uplink channel bandwidth. The uplink bandwidth allocation algorithm of EPON could be divided into the FBA and DBA. Because FBA allocate ONU for fixed size bandwidth (time slot) each time, so the statistical reuse could not be used among ONUs, and it could not accord with the abruptness of the network operation, and the bandwidth use rate is low. But DBA has not these disadvantages, and it could not only fairly allocate the uplink bandwidth, but provide better QoS support. At present, most research works emphasize particularly on the intensive DBA algorithm, i.e. OLT accepts the bandwidth request of ONU, and compute DBA, and allocate the uplink transmission window for ONU. Many articles only improved this intensive DBA algorithm, and emphasize the improvement of the bandwidth use rate, the time delay, and QoS. In this article, a 
new distributed scheduling algorithm is proposed, and it could fairly and effectively allocate the bandwidth. In this algorithm, the access control of the uplink channel is intensive, because it still could be run in OLT, but for all ONUs distributed in PON in the scheduling process, ONUs would decide the size of the uplink transmission window themselves.

\section{Distributed DBA algorithm}

\subsection{Basic principle}

The common character of the existing distributed DBA algorithms is that the computation of the uplink transmission time slot is accomplished by ONU, not by OLT, so ONU must know the transmission window information of other ONU. Therefore, the distributed DBA algorithm proposed before modified the frame of EPON. For example, in E. Wong's article (E. Wong, 2005, P.3) the network frame is simulated in LAN and all ONUs are conneted with one star coupler by two fibers, and ONUs could accept the frames transmitted by other ONUs. SR. Sherif (SR. Sherif, 2004, P.2483-2497) changes the light branch in EPON by a star coupler, so part of signal lights come from ONU could be reflected, and other ONUs will detect them. The DDBA algorithm proposed in this article needs not modify the existing EPON frame, and only transfer the window information transmitted by other ONUs to certain one ONU by the agency of OLT, and it completely accords with the MPCP protocol regulated by IEEE 802.3ah standard, and only needs add some new fields in the REPORT frame and the GATE frame.

In the algorithm of DDBA, ONU decides the size of tile slot, and transmits the REPORT frame to OLT, and after OLT receives the REPORT frame, it transmits it to the GATE frame at one, and the authorization length is the time slot requested by ONU. Of course, OLT adds some extra information in the GATE frame, so ONU could compute the size of the uplink transmission window. Extra information includes a weight vector $(\theta)$, i.e. the weight factors of all ONUs. These weight factors are defined as the proportion of the total using bandwidth allocated to the bandwidth of ONU and one polling cycle. ONU could know the size of other ONUs' transmission windows in last polling cycle by the weight information. When ONU transmits the REPORT frame, it will re-compute the weight factor according to the request bandwidth, and fill in relative field of the REPORT frame, and the weight information from OLT will be updated continually. When the scheduling begins, a fixed weight will be allocated to each ONU according to the information of SLA.

The polling mode generally includes the interleaved polling and the interleaved polling with pause. In the intensive DBA algorithm, if the interleaved polling mode is adopted, OLT will implement the DBA operation according to the REPORT algorithm of each single one ONU, and if the interleaved polling mode with pause is adopted, OLT will allocate the bandwidth after receiving the REPORT frames of all ONUs. Therefore, the former mode makes against that OLT makes fair and reasonable authorization, but the latter mode could ensure the justice of the bandwidth allocation under certain condition, i.e. the bandwidth use rate will be reduced, because the time slot will occur in two times of polling, and in this period, the uplink channel could not be utilized. For DDBA, because ONU could acquire the window information transmitted by other ONUs, and OLT needs not wait the arrival of all REPORT frames, so the interleaved polling mode is adopted (seen in Figure 1).

\subsection{Bandwidth allocation}

Here, $W_{M A X}$ is the maximum bandwidth (time slot) which could be allocated in one polling cycle. One cycle is the time expensed by OLT when it polls all ONUs, i.e. the sum of $\mathrm{N}$ time slots and the protection time slots among them. From the formula (1), when the sum of all ONU's weight factors is 1, the bandwidth of the i'th ONU is $\theta_{i} W_{\text {MAX }}$.

The concrete approach of the DDBA algorithm could be described as follows.

(1) OLT maintains one weight vector table (seen in Table 1), where $\theta_{i}(n)$ denotes the weight factor of the i'th ONU in the n'th cycle. When OLT receives the REPORT frame of the $i^{\prime}$ th ONU, it will compare the new weight factor and the factors in the weight vector table, and if the new weight factor is bigger, it will be used to update the value in the weight vector table. Then, OLT transmits the GATE frame to ONU, and allocates the authorization bandwidth in next cycle, and the value of bandwidth is the size of the REPORT frame requested by ONU. If the EPON system supports QoS, the GATE will carry the follow parameter matrix.

$$
\theta(n)=\left[\begin{array}{cc}
\theta_{11}(n) & \theta_{1 M}(n) \\
\theta_{N 1}(n) & \theta_{N M}(n)
\end{array}\right]
$$

Where, $\mathrm{N}$ denotes the amount of ONU, and $\mathrm{M}$ denotes the amount of QoS sort. In the description of algorithm, 
the value of M is 1. But the REPORT frame only carry the weight of single ONU and the information of QoS.

(2) When ONU receives the GATE frame, it will transmit the data with the length of $R_{i}(n)$ to OLT after the authorization time begins. Where, $R_{i}(n)$ denotes the bandwidth requested by ONU in the i'th cycle. Then ONU will compute the maximum transmission window in $n+1$ cycles according to the extra information in the GATE frame, and the computation formula is

$W_{i}(n+1)=\frac{\theta_{i}}{\sum_{j=1}^{N} \theta_{j}(n)} W_{M A X}$

Hereby, the size of the bandwidth requested by ONU in $\mathrm{n}+1$ cycles, i.e. $R_{i}(n+1)=\operatorname{MIN}\left(W_{i}(n+1), Q_{i}\right)$, where, $Q_{i}$ denotes the queue length of the i'th ONU. Finally, ONU transmits the REPORT frame at the end of the authorization time slot, and informs OLT its queue length and new weight factor. These weight factors could be obtained by the following formula.

$$
\theta_{i}(n+1)=\frac{R_{i}(n+1) \sum_{j=1}^{N} \theta_{j}(n)}{W_{M A X}}
$$

Thus, the authorization to the i'th ONU is accomplished, and other ONUs could be authorized according to above process. The beginning of the authorization time is still controlled by OLT, because only OLT know the size of the window transmitted by each ONU.

\section{Simulation and analysis}

The IPACT proposed by G. Kramer et al is an intensive interleaved polling algorithm, and its disadvantages have been described as follows. But the DDBA algorithm is a distributed interleaved polling algorithm, it could not only ensure the bandwidth use rate theoretically, but guarantee the justice of the bandwidth allocation. Here, these two algorithms will be simulated and compared. The IPACT algorithm adopts the limitation service mode to confirm the size of the transmission window, i.e. if the size of the bandwidth requested by ONU doesn't exceed the pre-established maximum transmission window, the size of the authorized time slot is the bandwidth requested by ONU, or else, the authorized length is this pre-established value.

By adopting the OPNET network simulation tool to model the EPON system, the network-level model is seen in Figure 2. The model adopts the tree network topology with one OLT and 16 ONUs. The distance from OLT to the light branch is $5 \mathrm{~km}$, and the distance from ONU to the branch is in $0-15 \mathrm{~km}$, so RTT will be distributed in 50 us to 200us evenly. Each ONU node meets with one user node which will produce the network operation, and transmit it to ONU.

To acquire real network performance, the operation model with self-similarity and correlation is adopted (M. Crovella, 1996). The Hurst parameter with the self-similarity source is 0.8 . According to the definition of Ethernet frame format, the lengths of the data package are in 512 bits to 12144 bits and obey the even distribution.

These concrete simulation parameters include that the speed from the user to ONU is $100 \mathrm{Mbps}$, and the uplink and downlink channel speed is $1 \mathrm{Gbps}$, and one buffer queue is set at the place of ONU, and the maximum length is $1 \mathrm{Mbyte}$, and the protection time slot is $2 \mathrm{~ms}$. To simulate the performance of the simulation algorithms in different referring loads, ONU will share the total loads evenly. For example, if the total referring load is $1 \mathrm{Gbps}$, the load referred by each ONU will be $62.5 \mathrm{Mbps}$, and the operation production speed is 9383 packets/s.

The statistical quantities collected by the simulation include the average end-to-end time delay, the average queue length, the uplink bandwidth use rate, and the downlink bandwidth use rate. The average end-to-end time delay includes three aspects, (1) the polling time delay, i.e. the time interval from time when the packet arrives at ONU to the time that ONU transmits the REPORT frame, (2) the authorization time delay, i.e. the time interval from the time that ONU transmits the request to the time that ONU receives the authorization, (3) the queue time delay, i.e. the awaiting time of the data package in the transmitter queue. The average queue length denotes the lengths of all data package in the ONU buffer queue, and the unit is bit. The downlink bandwidth use rate denotes the ratio of the bandwidth consumed by the control frame and the total bandwidth.

Under different referring loads, the performance data (seen in Figure 3) of IPACT and DDBA could be obtained after 3 seconds simulation. The abscissa denotes the referring loads which are standardized by the uplink channel speed 1 Gbps. When the abscissa is 0.7 , it denotes that the whole network referring load is $700 \mathrm{Mbps}$. From the 
Figure 3(a), when the total referring load is less than 0.75 , the average end-to-end time delays of two algorithms are almost same, but when the total referring load exceeds 0.8 , the average end-to-end time delay of the DDBA algorithm is a little less than the time delay of the IPACT algorithm. Especially when the load is 1, the time delay of the IPACT algorithm is more $11 \mathrm{~ms}$ than the time delay of the DDBA algorithm. At the location of ONU, the change curve of the average queue length is consistent with the change curve of time delay (seen in Figure 3 (b)), and after the load exceeds 0.8 , the queue lengths of two algorithms will increase quickly, but the increase of the queue length of the DDBA algorithm is slower. From the Figure 3 (c), when the load exceeds 0.9 , the use rate of the DDS algorithm to the uplink bandwidth will be kept above $90 \%$, but the IPACT algorithm only has about $88 \%$ bandwidth use rate. That could indicate the cause that the average end-to-end time delay and the average queue length under high load will increase abruptly, and the use of the uplink bandwidth will achieve the saturation. For the use rate of the downlink bandwidth, two algorithms could obtain almost same result, and only when the load exceeds 0.9 , the downlink bandwidth consumed by the DDBA algorithm will be less.

\section{Conclusions}

Aiming at the uplink channel access of the EPON system, a new distributed dynamic bandwidth allocation algorithm is proposed in this article. By the extra information transmitted by OLT, various ONU could decide the size of the uplink transmission windows based on its buffer queue length and other ONU bandwidth request information. The operation process of DBA is distributed in various ONUs, and OLT only assists it. The simulation result shows that under high network load, comparing with the IPACT algorithm, this algorithm could acquire lower end-to-end time delay and smaller average queue length.

\section{References}

E. Wong, and C. Chang-Joon. (2005). Efficient dynamic bandwidth allocation based on upstream broadcast in Ethernet passive optical networks. Optical Fiber Communication Conference. No.6. P.3.

G. Kramer, B. Mukherjee and G. Pesavento. (2002). Interleaved Polling Distribution Scheme in an Optical Access Network. Journal Photonic Network Communications. No.4(1). P.89-107.

M. Crovella and A. Bestavros. (1996). Self-similarity in World Wide Web traffic: Evidence and possible causes. In Proceedings of ACM SIGMETRICS International Conference on Measurement and Modeling of Computer Systems. Philadelphia PA, May 1996.

SR. Sherif, A. Hadjiantonis, G. Ellinas, et al. (2004). A novel decentralized Ethernet-based PON access architecture for provisioning differentiated QoS. Journal of Lightwave Technology. No.22. P.2483-2497.

Table 1. Weight vector table

\begin{tabular}{|l|l|l|l|l|}
\hline$O N U_{1}$ & $\mathrm{ONU}_{2}$ & $O N U_{3}$ & $\ldots$ & $O N U_{N}$ \\
\hline$\theta_{1}(n)$ & $\theta_{2}(n)$ & $\theta_{3}(n)$ & $\ldots$ & $\theta_{N}(n)$ \\
\hline
\end{tabular}

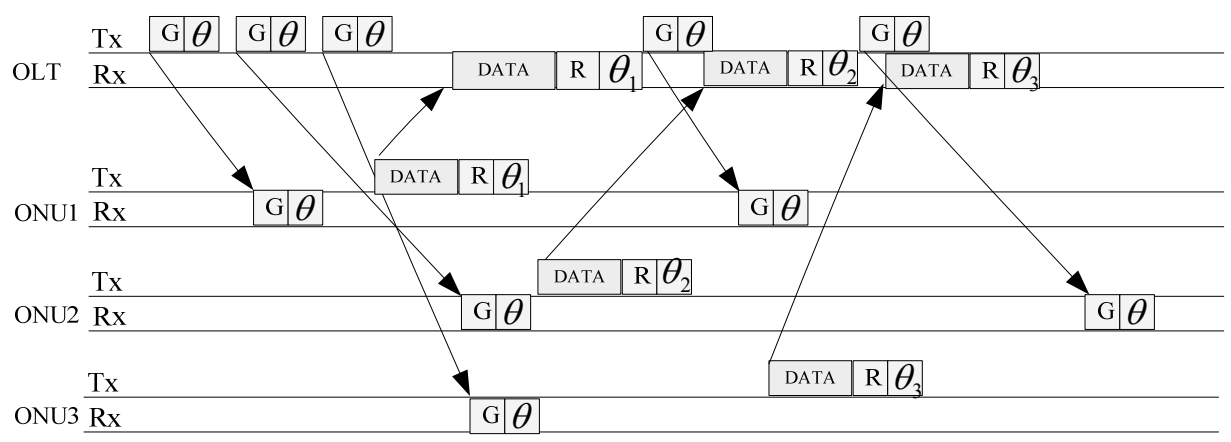

Figure 1. Interleaved Polling Mechanism of DDBA 


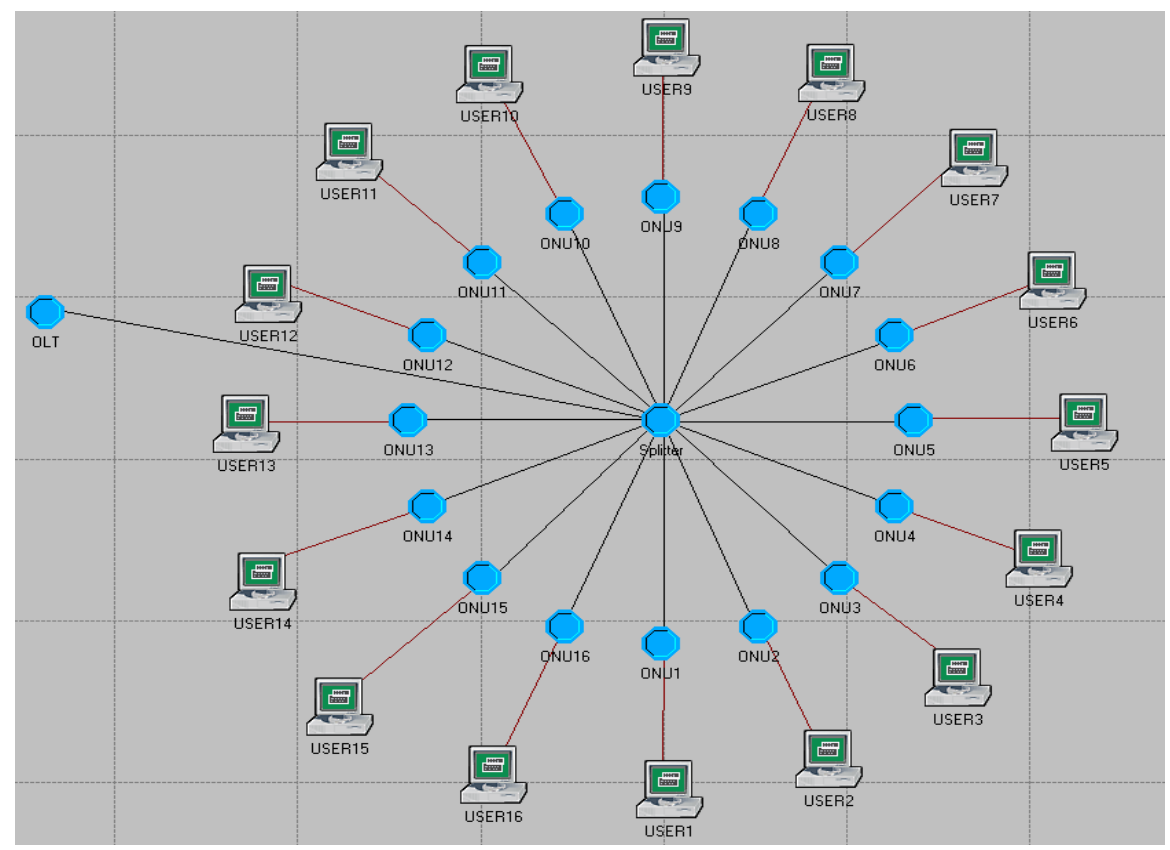

Figure 2. Network Model of EPON
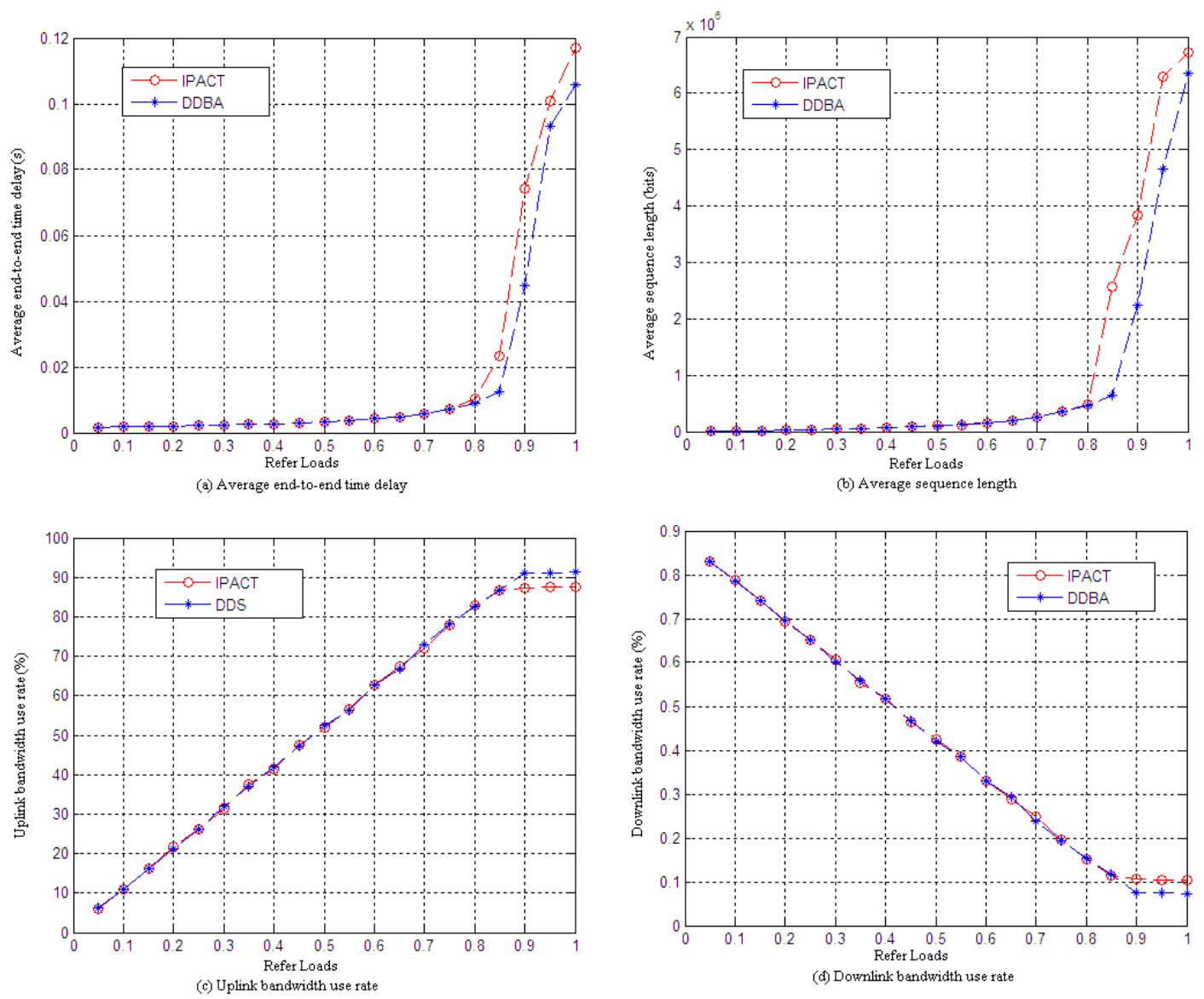

Figure 3. Performance Comparison of DDBA and IPACT 